\title{
The Fate of Massive Closed Strings
}

\author{
Bin Chen*, Miao $\mathrm{Li}^{\dagger}$ and Jian-huang She ${ }^{\dagger}$ \\ *Department of Physics, Peking University, Beijing 100871, P.R. China \\ ${ }^{\dagger}$ Institute of Theoretical Physics, Chinese Academy of Science, P.O.Box 2735, Beijing 100080, P.R. China
}

\begin{abstract}
We calculate the semi-inclusive decay rate of an average string state with toroidal compactification in the the superstring theory. We also apply this calculation to a brane-inflation model in a warped geometry and find that the decay rate is greatly suppressed if the final strings are both massive and enhanced for massless radiation.
\end{abstract}

\section{INTRODUCTION}

Our study of the massive closed string states was motivated by the renewed interests in cosmic string[1, 2, 3], and also by a desire to understand the mysterious open/closed duality in tachyon condensation [4, 5]. The revival of the cosmic string resides in the fact that in the low-energy models the cosmic strings could be created after inflation. In a class of string-inspired models on inflation, the tachyon condensation plays a fundamental rule. It has been shown that some strings, including fundamental string and solitonic strings, could be created after tachyon condensation. They are sufficiently stable and a few of them may survive and be observable in near future through gravitational wave detectors such as LIGO and LISA. Furthermore, in the decay products of the tachyon condensation, there are very massive closed string states, which could be an origin of the cosmic string. However these massive fundamental string states are not stable and may decay to other massive or massless states. In order to understand the fate of a typical massive F-string state better, it is essential to study its decay systematically. On the other hand, even without considering its phenomenological implication, the study of the decay of a typical massive string state is an interesting issue on its own right in string theory. To understand the tachyon condensation and the mysterious open/close string duality, the study of the fate of these massive string states is necessary.

The decay of massive strings is an old problem of string theory and it is generally difficult to extract detailed knowledge from conventional calculations mainly due to the exponentially growing state density. States at the same mass level may be very different, especially their decay properties. Fortunately in a realistic situation, such as the rolling tachyon case, details of string states are not important, and we are only interested in some sort of averaged decay rates. In an algebraic approach, J.L Manes considered in [8] the emission spectrum of the strings of all mass levels for bosonic (open and closed) strings. It was found there that the decay rates are universal, in the sense that the leading terms depend only on the mass level of the strings involved, and the more detailed properties of the states affect only the sub-leading terms.

To study the stringy properties relevant for cosmology, we need to consider the situation where some of the space-time dimensions are compactified. And in the realistic models, the superstrings rather than the bosonic strings are used. Therefore we will try to study the closed string decay in superstring in the flat spacetime with compactifications and then in a realistic model[ 6$]$ with warped compactification. The work reported here is based on the paper[7].

\section{CLOSED STRING DECAY: WITH COMPACTIFICATION}

We firstly study the emission spectrum of massive closed bosonic strings in the case where $d_{c}$ out of the total $D$ dimensions are compactified on a flat torus. A closed string state is characterized by its KK momentum numbers $n_{i}$, winding numbers $w_{i}$, and its mass $M$ and momentum $k$ in the noncompact dimensions. The mass shell condition reads

$$
\alpha^{\prime} M^{2}=4\left(N_{R}-1\right)+\alpha^{\prime} Q_{+}^{2}=4\left(N_{L}-1\right)+\alpha^{\prime} Q_{-}^{2}
$$

where $Q_{ \pm}=\sum \frac{n_{i}}{R_{i}} \pm \frac{w_{i} R_{i}}{\alpha^{\prime}}$, and $i=1, \ldots, d_{c}$.

Consider the process

$$
\left(M, n_{i}, w_{i}\right) \rightarrow\left(m, n_{1 i}, w_{1 i}\right)+\left(M_{2}, n_{2 i}, w_{2 i}\right)
$$

In the following, we set $\alpha^{\prime}=\frac{1}{2}$. 
We are interested in the averaged semi-inclusive twobody decay rate: for the initial string and one of the two final strings, we average over all states of some given mass, winding and KK momentum. Only the other string's state is fully specified (by keeping explicit its vertex operator). This decay rate can be written as

$$
\Gamma_{\text {semi-incl }}=\frac{A_{D-d_{c}}}{M^{2}} g_{c}^{2} \frac{F_{L}}{\mathscr{G}\left(N_{L}\right)} \frac{F_{R}}{\mathscr{G}\left(N_{R}\right)} k^{D-3-d_{c}} \prod_{i}^{d_{c}} R_{i}^{-1}
$$

with closed string coupling $g_{c}$, compactification radius $R_{i}$, and $F_{L}$ and $F_{R}$ are given by

$$
\begin{aligned}
& F_{L}=\sum_{\Phi_{i}} \sum_{\Phi_{f}}\left|\left\langle\Phi_{f}\left|V_{L}\left(n_{1 i}, w_{1 i}, k\right)\right| \Phi_{i}\right\rangle\right|^{2} \\
& F_{R}=\sum_{\Phi_{i}} \sum_{\Phi_{f}}\left|\left\langle\Phi_{f}\left|V_{R}\left(n_{1 i}, w_{1 i}, k\right)\right| \Phi_{i}\right\rangle\right|^{2},
\end{aligned}
$$

which can be written as

$$
F_{L}=\oint_{C} \frac{d z}{z} z^{-N_{L}} \oint_{C^{\prime}} \frac{d z^{\prime}}{z^{\prime}} z^{\prime-N_{2 L}} \operatorname{Tr}\left[z^{\hat{N}} V_{L}^{\dagger}(k, 1) z^{i \hat{N}} V_{L}(k, 1)\right] .
$$

In the following, we define $w=z z^{\prime}$, and $v=z^{\prime}$.

Note that

$$
\begin{aligned}
& \int d^{D} p \operatorname{Tr}\left[z^{L_{0}} V^{\dagger}(k, 1) z^{L_{0}} V(k, 1)\right] \\
= & f(w)^{2-D}\left(\frac{-2 \pi}{\ln w}\right)^{D / 2}<V^{\dagger}(k, 1) V(k, v)>_{c},
\end{aligned}
$$

where

$$
f(w)=\prod_{n=1}^{\infty}\left(1-w^{n}\right)^{2-D},
$$

and $\langle\cdots\rangle_{c}$ denote the correlators on the cylinder. Thus the masterformula for the amplitude squared is

$$
\begin{aligned}
F_{L} & =\oint_{C_{w}} \frac{d w}{w} w^{-N_{L}} f(w)^{2-D} \mathscr{I}_{N_{L}-N_{2 L}}(w) \\
\mathscr{I}_{n} & =\oint_{C_{v}} \frac{d v}{v} v^{n}<V_{L}^{\prime \dagger}\left(k_{L}, 1\right) V_{L}^{\prime}\left(k_{L}, v\right)>_{c}
\end{aligned}
$$

where the contours satisfy $|w|<|v|<1$, for $|z|,\left|z^{\prime}\right|<1$.

The key point here is that there exist a recursion relation[8],

$$
\mathscr{I}_{n+m_{L}^{2}}(w)=w^{n+m_{L}^{2}}\left[\mathscr{I}_{n}(w)+n+\frac{m_{L}^{2}}{2}\right],
$$

which allow us to write the $\mathscr{I}_{n}$ into a series summation. Straightforwardly, the master formula could be written as

$F=\sum_{p=1}^{A}\left(n-m_{L}^{2}\left(p-\frac{1}{2}\right)\right) \mathscr{G}\left[N_{L}-n p+\frac{1}{2} m_{L}^{2}\left(p^{2}-p\right)\right]+F_{N U}$ where $n=N_{L}-N_{2 L}$, and

$$
F_{N U}=\oint_{C_{w}} \frac{d w}{w} w^{-N} f(w)^{2-D} \mathscr{I}_{v}(w) w^{v A+\frac{1}{2} m_{L}^{2}\left(A^{2}+A\right)}
$$

and the generating function for the mass level density

$$
\operatorname{Tr} w^{\hat{N}}=f(w)^{2-D}=\sum_{N=0}^{\infty} \mathscr{G}(N) w^{N} .
$$

has been used.

In $F$, all terms except the last one are universal, in the sense that they does not depend on the details of any of the three string states involved. The last term $F_{N U}$ is non-universal. The contribution from $F_{N U}$ to $F$ is generically negligible, in comparison with the universal part contribution, except the case $A=0$ which happens when the emitted states carry a large fraction of the total mass.

One important observation is that we have inequality

$$
\sqrt{N_{1 L}}+\sqrt{N_{2 L}} \leq \sqrt{N_{L}}
$$

The equality saturate when

$$
k=0, \quad \frac{M}{Q_{-}}=\frac{M_{2}}{Q_{2-}},
$$

where $k$ is the momentum in the noncompact directions and $M_{2}, Q_{2}$ are the quanta of the outgoing string states with fixed level $N_{2 L}$. The same inequality holds in the right-mover. Thus if generically $N_{L}>N_{2 L}>\frac{N_{L}}{3}$, the first term dominates the whole summation in $F_{L}$, and the other terms will be neglected to get

$$
F_{L} \approx\left(N_{L}-N_{2 L}-\frac{1}{2} m_{L}^{2}\right) \mathscr{G}\left(N_{2 L}\right) .
$$

$F_{R}$ can be carried out in the same way.

We can get the total decay rate for decays into arbitrary states of given mass, winding and $\mathrm{KK}$ momentum by simply multiplying by the state density $\mathscr{G}\left(N_{1}\right)$

$$
\begin{aligned}
& \Gamma\left[\left(M, n_{i}, w_{i}\right) \rightarrow\left(m, n_{1 i}, w_{1 i}\right)+\left(M_{2}, n_{2 i}, w_{2 i}\right)\right] \\
\approx & A_{D-d_{c}} \frac{g_{c}^{2}}{M^{2}} \mathscr{N}_{L} \mathscr{N}_{R} \mathscr{G}_{L} \mathscr{G}_{R} k^{D-3-d_{c}} \prod_{i}^{d_{c}} R_{i}^{-1},
\end{aligned}
$$

where

$$
\mathscr{N}_{L}=N_{L}-N_{2 L}-\frac{1}{2} m_{L}^{2}, \quad \mathscr{N}_{R}=N_{R}-N_{2 R}-\frac{1}{2} m_{R}^{2},
$$

and

$$
\mathscr{G}_{L}=\frac{\mathscr{G}\left(N_{1 L}\right) \mathscr{G}\left(N_{2 L}\right)}{\mathscr{G}\left(N_{L}\right)}, \quad \mathscr{G}_{R}=\frac{\mathscr{G}\left(N_{1 R}\right) \mathscr{G}\left(N_{2 R}\right)}{\mathscr{G}\left(N_{R}\right)} .
$$

As long as $N_{1 L} \gg 1$ and $N_{2 L} \gg 1$, we can write

$$
\mathscr{G}_{L} \sim\left(2 \pi T_{H}\right)^{-\frac{D-1}{2}}\left(\frac{N_{1 L} N_{2 L}}{N_{L}}\right)^{-\frac{D+1}{4}} e^{-\sqrt{2} t_{L} / T_{H}},
$$


with the Hagedorn temperature $T_{H}=\frac{1}{\pi} \sqrt{\frac{3}{D-2}}$ and

$$
t_{L}=\sqrt{N_{L}}-\sqrt{N_{1 L}}-\sqrt{N_{2 L}} .
$$

We have in the above restored the multiplicative constant in front of the state density.

From the inequality above, we know that the dominant decay channel is the original incoming string break into two strings with the same

$$
\alpha=\frac{M}{Q_{-}}=\frac{m}{Q_{1-}}=\frac{M_{2}}{Q_{2-}}
$$

and without releasing any kinetic energy $k=0$.

The total decay rate is

$$
\begin{aligned}
& =\sum_{N_{2}} \sum_{\left(n_{1 i}, n_{2 i}\right)} \sum_{\left(w_{1 i}, w_{2 i}\right)} \Gamma\left[\left(M, n_{i}, w_{i}\right) \rightarrow\left(m, n_{1 i}, w_{i}\right), n_{i}, w_{i}\right) \\
& \text { he decay rate can be well approximately factorizze parts } \\
& \qquad \Gamma_{m}\left(M, n_{i}, w_{i}\right)=\Gamma^{k} \sum_{\left(n_{1 i}, n_{2 i}\right)} \mathscr{G}^{K K} \sum_{\left(w_{1 i}, w_{2 i}\right)} \mathscr{G}^{w},
\end{aligned}
$$

where we make saddle point approximation for the noncompact momentum, that is we let $k=0$, or $M_{2}=M-m$, when considering the KK modes and winding modes.

In general, the contribution from the KK-modes and windings could be just a constant factor and the dependence on the momentum $k$ could be encoded in

$$
\left(\frac{m M_{2}}{4}\right)^{2} \exp \left(-\left(\frac{\alpha}{\sqrt{\alpha^{2}-1}}+\frac{\beta}{\sqrt{\beta^{2}-1}}\right) \frac{k^{2}}{2 T_{H}} \frac{M}{2 m M_{2}}\right),
$$

where $\beta=\frac{M}{Q_{+}}$. Note that if we measure the energy of the one of the decay string with mass $m$, we find a temperature:

$$
T_{m}=\frac{T_{H}}{2}\left(\frac{\alpha}{\sqrt{\alpha^{2}-1}}+\frac{\beta}{\sqrt{\beta^{2}-1}}\right)^{-1}\left(1-\frac{m}{M}\right) .
$$

This reflects both the recoil effect and the influence of the KK-modes and windings.

It is remarkable that when $\alpha=1$ or $\beta=1$, the decay is completely suppressed. This interesting limit corresponds to the BPS condition $M=Q_{-}$or $M=Q_{+}$in the superstring case. As we show below, all the discussion here could be applied to the superstring. Therefore, the fact that the BPS string states are stable is reflect in the above special limit. Actually, the suppression not only happens in the decay to the final massive strings, it also happens in the thermal radiation of the massless particles. Moreover, from above, the decay of the near BPS string states are greatly suppressed.
Taking into account of the measure of integral, we have

$\Gamma^{k}=A_{d_{c}}^{\prime} g_{c}^{2}\left(2 \pi T_{H}\right)^{-D+1} \frac{M}{m} m_{R}^{-\frac{D-1+d_{c}}{2}} \int_{0}^{\infty} t^{\frac{D-3-d_{c}}{2}} e^{-\frac{t}{T_{H}}} d t$,

where

$$
t=\left(\frac{\alpha}{\sqrt{\alpha^{2}-1}}+\frac{\beta}{\sqrt{\beta^{2}-1}}\right) \frac{k^{2}}{2 T_{H}} \frac{M}{2 m M_{2}}
$$

and $m_{R}=\frac{m(M-m)}{M}$, and $A_{d_{c}}^{\prime} \approx$ const. $\prod_{i=1}^{d_{c}} R_{i}^{-1}$. Thus including the phase space factor, we have a MaxwellBoltzmann distribution for $t$, which could be taken as a measure of the total kinetic energy.

The mean kinetic energy released per decay could be characterized by

$$
<t>=\frac{D-1-d_{c}}{2} T_{H},
$$

which is independent of the masses and satisfies the equipartition principle in $D-1-d_{c}$ noncompact spatial dimensions.

Carrying out the integral, which is just a $\Gamma$-function, we get

$$
\Gamma^{k} \approx A_{D}^{\prime} g_{c}^{2} \frac{M}{m} m_{R}^{-\frac{D-1+d_{c}}{2}},
$$

with $A_{D}^{\prime}=$ const $. A_{d_{c}}^{\prime} T_{H}-\frac{\left(D-1+d_{c}\right)}{2}$.

The emission rate of strings of mass $m$ is

$$
\frac{d \Gamma(m)}{d m}=\frac{d \Gamma^{k}(m)}{d m} \sum_{\left(n_{1 i}, n_{2 i}\right)} \mathscr{G}^{K K} \sum_{\left(w_{1 i}, w_{2 i}\right)} \mathscr{G}^{w}
$$

where

$$
\frac{d \Gamma^{k}(m)}{d m}=\Gamma^{k} \rho(m)=A_{D}^{\prime \prime} g_{c}^{2} M m_{R}^{-\frac{D-1+d_{c}}{2}},
$$

with $A_{D}^{\prime \prime}=\frac{1}{4} A_{D}^{\prime}$. It can easily be checked that if we set $d_{c}=0$, the above result reduces to the flat space case.

Next let us consider the decay rate of a typical massive type II string state in the 10-d dimensional Minkowski space. The basic quantity to compute is one piece of the semi-inclusive amplitudes squared,

$$
F=\frac{1}{\mathscr{G}(N)} \sum_{\Phi_{i}} \sum_{\Phi_{f}}\left|\left\langle\Phi_{f}|V(k)| \Phi_{i}\right\rangle\right|^{2} .
$$

One observation is that NS emission is the dominant decay channel for $N$ large, and $\mathrm{R}$ sector is suppressed by $\frac{1}{N}$, with $N$ being the initial string mass level. For the NS channel, the key point is to check whether there is still a recursion relation when the fermions are included. The above tree level trace could still be converted to a correlator on the cylinder. What is new for the superstring 
case is that due to the presence of fermions, the correlator is a summation over spin structures. The final result is that we have the same recursion relation for $\mathscr{I}_{n}$ as in the bosonic string and the only change is to replace spacetime dimension 26 by 10 . Consequently, the study on the decay of the massive string states in the bosonic string theory with toroidal compactification could be carried over to the superstring case directly.

\section{A REALISTIC MODEL}

The results on the toroidal compactifications can be used to estimate the massive string decay rates in the more realistic KKLMMT model, where the type IIB strings live in a highly warped throat with Klebanov-Strassler geometry.

A general warped geometry

$$
d s^{2}=H^{-\frac{1}{2}}(Y) \eta_{\mu v} d X^{\mu} d X^{v}+H^{\frac{1}{2}}(Y) g_{i j} d Y^{i} d Y^{j}
$$

will induce a potential

$$
V(Y)=\frac{1}{2 \pi \alpha^{\prime} H^{\frac{1}{2}}(Y)}
$$

on the string worldsheet, this potential confines the string to a small region in the compact dimension near the position where this potential has a minimum. Fluctuations in these compact dimensions

$$
<Y^{i} Y^{i}>=\frac{\alpha^{\prime}}{2} \ln \left[1+\frac{1}{2 \pi \alpha^{\prime 2} H^{\frac{1}{2}}(0) \partial_{i}^{2} V(0)}\right]
$$

provide an effective compactification volume for strings living in. When $\partial_{i}^{2} V$ is small in string units, that is, the geometry varies slowly on the string scale, we can look upon the strings in such a warped geometry as in a box with the effective compactification volume, and the flat space calculations above applies.

For the warped geometry, what we have discussed represents results seen by a 10-dimensional observer. And the quantities measured by the 4-dimensional observer, in our case the mass and the momentum, get red-shifted. Thus we should multiply $M, m, M_{2}$ and $k$ all by a red-shift factor $H^{\frac{1}{4}}(0)$. Now the mass shell conditions read like

$$
\alpha^{\prime} M^{2} H^{\frac{1}{2}}(0)=4(N-1)
$$

The emission rate of a string with mass $m$ from a typical string of mass $M$ in the Klebanov-Strassler geometry can finally be estimated to be

$$
\frac{d \Gamma_{K S}(m)}{d m} \approx A_{K S} g_{c}^{2} H^{-\frac{13}{8}}(0) M m_{R}^{-\frac{15}{2}},
$$

with $A_{K S}=\frac{1}{16 \pi^{5}} \frac{V_{\min }}{V_{\perp}}$.

With $H^{-\frac{1}{4}}(0) \sim 10^{-4}$, we see that the emission rate is suppressed by a factor of order $10^{-26}$ in this warped geometry! Thus in this warped geometry, the decay channels for a massive string to two massive strings are greatly suppressed.

We note that the situation is different if one of the two final strings is massless. It is found that the decay rate is proportional to the mass of the initial string. So including the redshift factor, it can be written as

$$
\Gamma_{\text {massless }} \sim g_{c}^{2} M H^{\frac{1}{4}}(0),
$$

from which we see that this decay channel is enhanced by order $10^{4}$. Thus in such warped geometries, massive strings may lose their energy mainly through gravitational radiation.

\section{CONCLUSION}

We calculate the cross section of the decay of a massive closed string state into two massive closed string states; The decay shows some novel features: KK modes and windings proportional to the masses preferred; the superstring share the same recursion relation with the bosonic string; In a realistic model with warped geometry, the massless decay is enhanced by the redshift factor.

\section{Acknowledgments}

This research project was supported by a grant from CNSF and a grant from CAS. We would like to thank J.L Manes for valuable comments. BC would like to thank the invitation from PASCOS-05 to present this work.

\section{REFERENCES}

1. M.B. Hindmarsh and T.W. Kibble, Cosmic strings, Rept.Prog. Phys. 58, 477(1995); A. Vilenkin and E.P.S. Shellard, Cosmic strings and other topological defects, Cambridge Unv. Press (Cambridge 1994).

2. E. J. Copeland et.al., Cosmic F- and D-strings, hep-th/0312067, J. Polchinski, Introduction to Cosmic F- and D-strings, hep-th/0412244

3. G. Dvali et.al., D-term strings, JHEP 0401:035,2004, hep-th/0312005, G. Dvali and A. Vilenkin, Formation and evolution of cosmic D-strings, JCAP 0403:010,2004, hep-th/0312007

4. A. Sen, Rolling Tachyon, JHEP 0204 (2002) 048, hep-th/0203211 B. Chen et.al., Gravitational Radiation of Rolling Tachyon, JHEP 0211 (2002) 050, hep-th/0209222. N. Lambert et.al., Closed strings from decaying D-branes, hep-th/0303139

5. A. Sen, Open-closed duality at tree level, Phys. Rev. Lett. 91 (2003)181601, hep-th/0306137. D. Gaiotto et.al., 
Closed Strings as Imaginary D-branes, Nucl.Phys. B688 (2004) 70-100, hep-th/0304192

6. S. Kachru et.al., Towards inflation in string theory, JCAP 0310:013,(2003), hep-th/0308055

7. B. Chen, M. Li and J-H, She, The Fate of Massive F-Strings, JHEP 06 (2005)009, hep-th/0504040

8. J. L. Manes, Emission Spectrum of Fundamental Strings: An Algebraic Approach, Nucl. Phys. B621:37-61,2002 |hep-th/0109196|. 